Eurostudia

\title{
Affirmation religieuse, politique publique et alternance politique aux États-Unis
}

\section{Blandine Chélini-Pont}

Volume 13, numéro 1-2, 2018-2019

Cultures en contact, entre régulations et représentations

URI : https://id.erudit.org/iderudit/1064493ar

DOI : https://doi.org/10.7202/1064493ar

Aller au sommaire du numéro

Éditeur(s)

Le Centre canadien d'études allemandes et européennes

ISSN

1718-8946 (numérique)

Découvrir la revue

Citer cet article

Chélini-Pont, B. (2018). Affirmation religieuse, politique publique et alternance politique aux États-Unis. Eurostudia, 13(1-2), 157-177.

https://doi.org/10.7202/1064493ar
Résumé de l'article

Cet article s'intéresse au poids de la variable religieuse dans l'identité des partis républicains et démocrates depuis les années 1990 puis dans la politique publique qu'ils ont générée au gré du jeu politique au Congrès, entre Congrès et Président et que la Cour Suprême a approuvée ou invalidée. Nous verrons se dessiner clairement deux usages de la référence religieuse et deux types de politiques publiques presque aux antipodes. Nous nous interrogerons enfin sur l'avenir de cette opposition solidement construite, parce que sa logique risque bientôt de s'enrayer face à une réalité religieuse et démographique en pleine transformation.
Tous droits réservés ( Le Centre canadien d'études allemandes et européennes, 2019
Ce document est protégé par la loi sur le droit d'auteur. L'utilisation des services d'Érudit (y compris la reproduction) est assujettie à sa politique d'utilisation que vous pouvez consulter en ligne.

https://apropos.erudit.org/fr/usagers/politique-dutilisation/ 


\title{
Affirmation religieuse, politique publique et alternance politique aux États-Unis
}

\author{
Blandine Chélini-Pont \\ Université d'Aix-Marseille
}

\section{Résumé}

Cet article s'intéresse au poids de la variable religieuse dans l'identité des partis républicains et démocrates depuis les années 1990 puis dans la politique publique qu'ils ont générée au gré du jeu politique au Congrès, entre Congrès et Président et que la Cour Suprême a approuvée ou invalidée. Nous verrons se dessiner clairement deux usages de la référence religieuse et deux types de politiques publiques presque aux antipodes. Nous nous interrogerons enfin sur l'avenir de cette opposition solidement construite, parce que sa logique risque bientôt de s'enrayer face à une réalité religieuse et démographique en pleine transformation.

\section{Abstract}

This article is focusing on the religious variable in the Republican and Democratic parties' identity since the 1990s. The two parties generated contradictory public policies depending on the political game in the Congress, between the Congress and the President, and consequently on the Supreme Court's activity. Two uses of religious references and two types of public antagonistic policies will be drawn. Finally, the article will examine the future of this solidly constructed opposition, because its logic shall soon be updated, if the parties want to gain or to keep their voters in the religious and demographic change of the country. 
Si nous tentons une description simple et sans doute très imparfaite de la politique publique états-unienne, nous pourrions la présenter comme une somme mouvante résultant du jeu de concurrence entre la Présidence et le Congrès fédéral, lui-même agissant comme un miroir des luttes partisanes entre représentants et sénateurs des deux grands partis historiques. Cette politique publique met en scène des mobilisations antagonistes qui quasiment à chaque fois aboutissent à un compromis entre le Président et le Congrès. Le compromis reflète cependant la tendance majoritaire du moment. C'est donc par le cumul des initiatives législatives et de leurs aboutissements, par la matière des executive orders présidentiels, abondant ou au contraire concurrençant la volonté du Congrès quand celui-ci a changé de majorité, et par le contenu des batailles judiciaires arrivant jusqu'à la Cour Suprême, corrigeant ou accompagnant la production législative et exécutive, qu'on peut tenter de repérer la fabrique de la politique publique fédérale.

La variable religieuse, très présente dans la mise en forme à la fois des identités partisanes et des thèmes mobilisateurs, est une donne essentielle dans l'alternance des orientations publiques. Par elle, on peut affirmer qu'il existe aux Etats-Unis deux politiques publiques religieuses qui s'imbriquent et qui s'opposent (D'Antonio et al. 2013).

Cette pénétration de la variable religieuse s'explique pour trois raisons. Les partis véhiculent un «esprit » de la République américaine, invoquant l'une des deux faces de la narration constitutionnelle, la religieuse et la libérale. Le camp du Christian Establishment se dresse contre celui du Liberal Establishment, recoupant la droite et la gauche de manière schématique, même si les républicains défendent aussi les libertés et si les démocrates ne dénigrent pas l'importance de la religion. Le camp du Christian Establishment regroupe tous ceux qui défendent l'identité chrétienne de l’Amérique comme source de ses 
institutions, de ses valeurs collectives, de son droit, de sa morale (sexuelle), de son esprit public existant «depuis toujours». Ils correspondent à une forte sédimentation historique qui se maintient dans une bonne moitié de l'opinion américaine. De l'autre, les démocrates se considèrent comme les gardiens des Lumières anglaises et américaines. Ils défendent le culte des Pères fondateurs, les libertés privées et les droits égaux. Ils manifestent un éthos inclusif et plutôt déiste, et comprennent l'esprit de l'Amérique comme la coagulation heureuse du pluralisme communautaire. Deux devises caractériseraient ces camps : In God we trust d'un côté, E pluribus unum de l'autre.

Il existe ensuite une corrélation entre l'engagement religieux et l'engagement politique, quand la cause à défendre recoupe un impératif spirituel. Il en fût ainsi des combats pour ou contre l'abolition de l'esclavage, la tempérance, les droits des femmes, l'éducation publique, ou les droits civils. L'engagement religieux est aujourd'hui focalisé sur d'autres causes tout aussi mobilisatrices: l'avortement, l'homosexualité, la sécurité sociale, la justice économique, l'immigration, l'environnement. Les partis politiques, au-delà de leur sensibilité civique, épousent ou récupèrent ces causes.

Enfin, il existe une forme d'adéquation entre la répartition politique des élus au sein du Congrès et leur répartition religieuse. Selon la représentation que les partis projettent de la République américaine, chrétienne exceptionnelle d'un côté ou libérale inclusive de l'autre, leurs élus sont proches de telle ou telle dénomination religieuse, avec de grandes chances que leurs électeurs le soient eux aussi, car les causes dont nous avons parlé à l'instant vont recouper la représentation que les partis se font de la République. Quand nous regardons les appartenances religieuses des membres du Congrès américain pour la 116 législature, nous voyons se dessiner un partage assez net des appartenances religieuses des élus qui ressemble aux législatures précédentes. D'un côté, nous 
trouvons un bloc identitaire-républicain qui est aussi un bloc protestant-chrétien, soit 99,2 \% qui se déclarent chrétiens dont $69 \%$ protestants, 25,4\% catholiques, 3,2 \% mormons, $0,8 \%$ orthodoxes et juifs ${ }^{1}$. Face à ce bloc, l'autre bloc que nous avons défini comme pluraliste-démocrate est à la fois chrétien et «autre ». Ce bloc est en effet toujours majoritairement chrétien, mais il accueille toutes les minorités religieuses non représentées chez les républicains. 78,4\% se déclarent chrétiens, dont $41,5 \%$ protestants et 35,1\% catholiques - les deux groupes étant en diminution. Chez ces chrétiens un peu moins nombreux, le chiffre des nonaffiliés augmente : 10,3\% contre 3,3\% à la législature précédente. Il y a plus de juifs $(11,3 \%)$, plus de musulmans $(1,1 \%)$ et d'hindous $(1,1 \%)$, plus de bouddhistes $(0,6 \%)$ et d'unitariens $(0,6 \%)$ et enfin, grande première, le parti démocrate compte $6,4 \%$ de personnes sans religion soit exactement 6 fois plus que précédemment, pour zéro toujours chez les républicains.

Quand nous appliquons la répartition partisane à l'électorat lui-même, que constatons-nous? L'électorat protestant vote majoritairement pour les républicains, la diversité minoritaire-confessante et la population sécularisée/sans religion vote pour le Parti démocrate ${ }^{2}$. Quant à l'électorat catholique, il penche tendanciellement vers le Parti républicain depuis les années 1980, mais son vote alterne au moment des présidentielles : entre $50 \%$ et $52 \%$ pour Bush, entre $54 \%$ et $50 \%$ pour Obama. Lors des élections de 2016, les catholiques ont eu le vote le plus balancé du spectre: $48 \%$ pour la démocrate Clinton, $45 \%$ pour le républicain Trump et $7 \%$ pour les petits partis. Le pluralisme ethnoracial comme

\footnotetext{
1 "Faith on the Hill, The Religious Composition of the $116^{\text {th }}$ Congress », Pew Research Center, 3 janvier 2019; http://www.pewforum.org/2019/01/03/faith-on-the-hill-116/.

2 "How the Faithful Voted: A Preliminary 2016 Analysis ", Pew Research Center, 9 novembre 2016; http://www.pewresearch.org/fact-tank/2016/11/09/how-the-faithful-voted-a-preliminary-2016-analysis/.
} 
la forte sécularisation de cette confession particulière expliquent la variation $\mathrm{du}$ vote, trouvant des causes à rallier autant à droite qu'à gauche ${ }^{3}$.

Au gré des victoires électorales engrangées par un camp ou l'autre depuis les années 1990, nous pouvons vérifier le poids de la variable religieuse dans l'idée que les partis véhiculent de la République, puis son poids dans la décision publique, selon le jeu politique décrit plus haut. Nous verrons se dessiner clairement deux usages de la référence religieuse et deux types de politiques publiques aux antipodes, et devenus inflammables dans leur opposition. Pourtant, nous nous interrogerons sur l'avenir réel de cette alternance. La réalité démographique de la population américaine est marquée par une forte progression de l'irréligion, une forte progression de la population hispanique catholique, un déclin relatif du christianisme et une pluralisation religieuse croissante. De tels faits sociaux risquent de produire ensemble une corrosion du jeu traditionnel et une remise à plat des deux variantes de l'éthos public.

\section{L'éthos public défendu par les républicains : One Nation under God}

Les républicains n'auraient pu militer ces trente dernières années pour une République chrétienne américaine, si de multiples traces de son existence n'avaient été laissées en héritage dans le passé institutionnel des États-Unis : par exemple la journée nationale de prière instaurée par George Washington et maintenue jusqu'à nos jours. Ils ont cependant pris le parti d'activer cette mémoire sous un angle renouvelé par la droite religieuse au tournant des années 1970. Cette droite a très clairement réaffirmé la nature chrétienne des États-Unis comme la seule véritable, accusant l'évolution libérale des institutions d'être une

\footnotetext{
${ }^{3}$ Données recueillies par le Georgetown University's Center for Applied Research in the Apostolate (CARA), 31 mars 2017; https://twitter.com/caracatholic/status/847891169254277121; cité par Michael J. O'Laughlin, "New Data Suggest Clinton, Not Trump, won Catholic Vote », America. The Jesuit Review [en ligne], 6 avril 2017; https://www.americamagazine.org/politics-society/2017/04/06/new-data-suggestclinton-not-trump-won-catholic-vote.
} 
infidélité à leur esprit d'origine. Dans ce discours, les clauses du premier amendement des Etats-Unis ont été prises à témoin. Elles ont été relues pour démontrer qu'elles ne contenaient aucune once de neutralité, concept jurisprudentiel progressivement et logiquement dégagé par la Cour suprême, en contrepoids du concept de liberté religieuse, qui a permis de protéger les minoritaires de la discrimination et de l'obligation d'être soumis à un ordre public protestant.

Les deux clauses du premier amendement portant sur la place de la religion dans la République ont été investies par la droite religieuse américaine comme protégeant d'abord et avant tout une religiosité chrétienne préjugée commune à toute la population. Selon elle, la séparation, telle que Jefferson l'avait expliquée en son temps à des concitoyens baptistes, à savoir l'inexistence assumée de tout lien constitutionnel entre l'État, une ou plusieurs Églises, ne signifie pas la neutralité des institutions, mais la seule absence d'église établie. Selon elle toujours, le libre exercice du culte signifie exactement, depuis son introduction comme amendement, la liberté de religion - alors même que cette dernière a été un résultat de l'évolution jurisprudentielle. Les grandes décisions fédérales solidifiant le principe de neutralité comme Everson v. Board of Education (1947), Engel v. Vitale (1962) ou Lemon v. Kurtzman (1971) sont considérées par cette droite religieuse comme le fruit d'un sécularisme agressif, tandis que les jurisprudences qui ont péniblement construit le principe de liberté religieuse Cantwell v. Connecticut (1940). Reynolds v. US (1879). Connecticut v. Yoder (1972) sont, elles, oubliées alors qu'elles l'ont dégagé du Protestant Establishment qui a caractérisé les États fédérés, l'esprit des lois fédérales et la religiosité publique durant tout le XIXe siècle et la première partie du XXe siècle (Issaoui 2016).

La littérature conservatrice s'est développée depuis les années 1970 pour défendre l'esprit chrétien du public square, l'éthos chrétien de la République. La 
liberté religieuse - première des libertés - est le socle qui garantit aux citoyens américains d'être prioritairement respectés dans leurs convictions et leurs pratiques par la loi, même s'il faut pour cela la vider de sa portée générale pour protéger leurs croyances par exemption.

\section{La défense d'un esprit public chrétien}

Nous pouvons lier l'argumentaire ci-dessus présenté avec l'ensemble des initiatives légales, exécutives et judiciaires des républicains, de la période qui court des années 1990 à aujourd'hui, durant laquelle leur parti a eu le plus fréquemment la majorité du Congrès et la Présidence des États-Unis. Durant cette période, la sauvegarde de l'esprit chrétien de la République a pris plusieurs visages, depuis la tentative de modification constitutionnelle ou fédérale, au changement législatif réussi et à la bataille judiciaire payante.

Parmi les tentatives d'amendement constitutionnel figure celle de la prière chrétienne à l'école, considérée dans les années 1960 comme une violation de la Constitution et arrêtée dans les écoles publiques. Ces tentatives se chiffrent à une bonne quinzaine entre 1994 et 2006. Un autre cas répété est celui de l'amendement affirmant la soumission de la Constitution à l'autorité de Dieu, amendement connu sous les noms de God ou Jesus amendement. Il a connu plus de 200 propositions depuis 1787 et une bonne dizaine dans les années 1990 et 2000.

Parmi les tentatives de lois fédérales religieuses, nous pouvons citer le projet du Constitution Restoration Act de 2005, particulièrement alambiqué, prétendant interdire de poursuivre toute entité fédérale, État, gouvernement local et leurs agents respectifs qui reconnaîtraient Dieu comme source souveraine de la Loi, de la Liberté et du Gouvernement ${ }^{4}$. Il faut également noter la poussée de

\footnotetext{
${ }_{4}$ S.520 Constitution Restoration Act of 2005 (119th Congress) : https://www.congress.gov/bill/109thcongress/senate-bill/520/text.
} 
témoignages fidéistes de la part de certaines cours de justice des États du sud, installant dans leur rotonde des sculptures représentant les Dix Commandements donnés par Dieu à Moïse pour son Peuple, ce qui a donné lieu à des procès fédéraux dont deux se sont conclus à la Cour suprême : cette dernière a ordonné leur retrait pour violation de la Constitution à l'intérieur des bâtiments, mais accepté leur présence dans les espaces verts extérieurs, en tant que décoration « culturelle $»^{5}$.

Il y a eu par ailleurs un certain nombre d'initiatives qui ont réussi. De manière très symbolique, le Congrès républicain a réaffirmé dans une résolution de 2002 le caractère sacré du serment au drapeau - instauré en 1953 par Eisenhower - avec l'assertion finale « One Nation under God». Cette résolution a répondu à l'affaire californienne dite Newdow, du nom de son instigateur militant associatif athée, plaidant l'inconstitutionnalité de ce serment d'allégeance. Quoiqu'ayant été jugée inconstitutionnelle par le Circuit fédéral de Californie, l'affaire ne fut pas jugée par la Cour suprême grâce à un vice de la plainte. Plus près dans le temps se situe la question des prières au début des conseils municipaux. Elle a été 'gagnée' par les républicains, après validation de cette pratique par la Cour suprême, en s'appuyant sur une jurisprudence antérieure de 1983 conservant la prière au Congrès avant les sessions ${ }^{6}$. La pratique de la prière publique pendant la cérémonie d'investiture du président des Etats-Unis est également bien vivace, tout comme celle du National Prayer Breakfast, instaurée de manière informelle par un groupe de congressistes républicains, The Fellowship, sous Eisenhower. Depuis, il est une mondanité obligatoire pour les Présidents. À l'édition de février 2018, Donald Trump y rappela que le peuple américain est

\footnotetext{
${ }^{5}$ Van Orden v. Perry (Texas) et McCreary County v. ACLU of Kentucky (2005).

${ }^{6}$ Maintien des prières du Congrès Marsh $v$. Chambers (1983) et des prières dans les conseils municipaux Town of Greece v. Galloway (2014).
} 
« une nation de croyants », les Etats-Unis un pays « renforcé par le pouvoir de la prière » et que Dieu doit être remercié « pour la bénédiction d'être Américain » ${ }^{7}$.

\section{La First Freedom}

L'année précédente, en 2017, le Président Trump avait centré son discours sur la grandeur de la liberté religieuse et la nécessité de sa défense. Il avait promis pendant la campagne présidentielle de faire abroger l'amendement Johnson, une disposition du code fiscal qui interdit aux Églises de soutenir financièrement un candidat politique dans une campagne ${ }^{8}$. Cette promesse tenue est une illustration de la pression qui s'exerce dans les rangs républicains, depuis les années 1990, pour investir la liberté religieuse comme objectif prioritaire.

Attisée par l'exemple contrariant de jurisprudences fédérales la limitant dans le cadre du travail'9, une bataille législative s'est engagée qui a été gagnée. Malgré l'échec répété de l'Istook amendement, présenté en 1997, 1998 et dans les années 2000, sommant le gouvernement fédéral de respecter toutes sortes de garanties pour les citoyens croyants ${ }^{10}$, les législatures majoritairement républicaines ont fait passer un certain nombre de lois fédérales pour favoriser le droit des croyants, en cas de confrontation avec d'autres lois fédérales. Une

\footnotetext{
${ }^{7}$ Gauthier Vaillant, « "Prayer Breakfast", Donald Trump face aux chrétiens », La Croix [en ligne], 8 février 2018; https://www.la-croix.com/Religion/Prayer-Breakfast-Donald-Trump-face-chretiens-2018-02-08$\underline{1200912359}$

8 Alexis Buisson, «Donald Trump veut laisser les Églises financer les candidats politiques », La Croix [en ligne], 13 février 2017; https://www.la-croix.com/Monde/Ameriques/Donald-Trump-veut-laisser-Eglisesfinancer-candidats-politiques-2017-02-13-1200824359. En 2017 le projet de loi fiscale présenté par le Comité sur les dotations de la Chambre des représentants, prévoyant la fin de cette interdiction, n'a pas été retenu.

9 US v. Lee (1982); Goldman v. Weinberger (1986); Employment Division v. Smith (1990).

${ }^{10}$ Cela veut dire : ne jamais empêcher les croyants de reconnaître Dieu selon le diktat de leur conscience, de reconnaître aux croyants leur droit de prier, de ne pas limiter leur héritage et tradition sur la propriété publique, incluant l'école, de ne jamais leur interdire d'inviter quiconque à rejoindre la prière ou des activités religieuses, de ne jamais les empêcher d'entamer des prières à l'école, bref de ne pas discriminer les personnes croyantes.
} 
révolution législative s'est dessinée qui a transformé les controverses autour de la défense de la liberté religieuse en défendant l'exception croyante face à la loi commune. Le Religious Freedom Restoration Act (RFRA, 1993) rend obligatoires le contrôle strict de l'intérêt impératif de l'État et l'usage de la solution la moins restrictive pour l'expression religieuse. La loi sur la protection de la liberté religieuse et des dons caritatifs, Religious Liberty and Charitable Donation Protection Act (1998), exclut les dons aux organismes religieux et caritatifs des recouvrements de créances et les intègre dans les dépenses autorisées par les mandataires en cas de faillite. Le Religious Land Use and Institutionalized Persons Act, également de 1998, garantit des protections accrues en faveur des groupes religieux et des personnes en matière foncière (privilèges fiscaux et de crédit, priorités d'achat et de vente) et en matière de respect des croyances religieuses dans le cadre des prisons et des hôpitaux psychiatriques.

Assouplissement du principe de non-subventionnement

Par ailleurs, le principe constitutionnel fédéral déduit du premier amendement de non-subventionnement public des religions a connu un très sérieux infléchissement. Après l'échec d'un projet de loi fédérale, poussé par une partie des républicains, le Faith-Based and Communities Initiatives Act, le Président Bush Jr. a décrété un ordre exécutif intitulé Faith-Based Initiatives en 2000 : les écoles privées et les institutions religieuses sont devenues éligibles à des financements publics. Attaqué, cet ordre exécutif n'a pas été considéré comme une violation constitutionnelle. Le Congrès républicain a par la suite voté avec succès des lois fédérales de la même veine: le Charity Aid, Recovery and Empowerment Act de 2002 en faveur d'œuvres sociales religieuses, puis le Scholarship for Opportunity and Results Act en 2004, pour financer des bourses payant les frais d'écoles religieuses aux enfants méritants du district fédéral. Le programme entraîna la création d'un Office of Faith-Based and Community 
Initiatives à la Maison-Blanche en 2007, qui n'a pas été inquiété par un recours intenté contre son existence. L'Office a été maintenu par le Président Obama et le programme SORA a été revoté en mars 2017 par le Congrès.

La vie et l'hétérosexualité du mariage comme God-given

La diminution de l'étanchéité financière de l'Etat fédéral vis-à-vis des activités religieuses sociales et éducatives est néanmoins passée largement inaperçue. Elle a été éclipsée par la grande bataille sur les questions de sexualité et de reproduction depuis les années 1970.

Dans cette « guerre culturelle», le mouvement Stop ERA (1972-1975) a été un point de départ, comme contre-feu à la jurisprudence Roe v. Wade de 1973, où la Cour suprême décriminalisa l'avortement, par le biais de la due process clause attachée au 14e amendement sur la protection de l'exercice de leurs droits par les citoyens américains. Ce mouvement, ancêtre de la droite religieuse, réussit par sa campagne acharnée à défaire le vote pourtant prévu comme consensuel de l'amendement fédéral sur l'égalité des droits entre homme et femme. Après cette victoire, le Hyde amendement, voté en 1976 et constamment renouvelé dans les lois sur la protection sociale, interdit le financement et remboursement de l'avortement par des fonds publics, à l'exception d'une grossesse issue d'un inceste et/ou d'un viol. Cet amendement est suivi d'une série de lois fédérales réduisant très sérieusement le périmètre de l'avortement comme élément du droit à la vie privée. Le Partial Birth Abortion Act est adopté par le Congrès en 2003, après une jurisprudence de la Cour suprême - Planned Parenthood v. Casey (2000) - jugée laxiste par les républicains. L'Unborn Victim of Violence Act est voté en 2004 et 38 États ont adopté par la suite des lois équivalentes. L'arrêt Gonzales v. Carhart de 2007 a été largement interprété comme un changement de cap dans la jurisprudence de la Cour suprême en faveur d'une restriction du droit à 
l'avortement, changement permis par le départ à la retraite de la juge Sandra Day O'Connor et son remplacement par le juge Samuel Alito.

Sur la question du mariage homosexuel, la bataille a commencé avec le Defense of Marriage Act, loi fédérale de 1996 dont la troisième section dispose que les bénéfices protecteurs respectifs du mariage ne peuvent concerner qu'un couple hétérosexuel. Comme cette loi a été immédiatement attaquée devant les juridictions ${ }^{11}$, le Congrès, à chaque majorité républicaine, a tenté par dix fois d'introduire un Federal Marriage Amendment définissant constitutionnellement le mariage comme une union entre un homme et une femme. Entre 1998 et 2012, à son exemple, 37 États se sont dotés d'un amendement constitutionnel par voie législative ou par référendum sur la nature hétérosexuelle du mariage. Plusieurs arrêts de la Cour suprême ont invalidé finalement ces amendements comme contraires au $14^{\mathrm{e}}$ amendement, sur la base de l'equal protection clause ${ }^{12}$. De même et pour l'instant, la Cour suprême n'a pas validé comme objection de conscience l'invocation de complicité de péché, invoquée par certains plaignants pour refuser de vendre, louer, ou commercer avec des couples homosexuels ${ }^{13}$.

\section{La fronde exemptionniste contemporaine}

Cependant, la limite est devenue fine. Les ingrédients d'une fronde exemptionniste ont été réunis quand Barack Obama a voulu porter devant le 111 Congrès, au temps d'une majorité démocrate à la Chambre, sa réforme de la santé, sous les noms de Patient Protection and Affordable Care Act et de Health Care and Education Reconciliation Act. La modération préalable de ces lois qui

\footnotetext{
${ }^{11}$ Pour finir par être déclarée inconstitutionnelle dans sa 3e section en 2013 car contraire au 5e et $14^{\mathrm{e}}$ amendements, dans l'arrêt United States v. Windsor(2013).

12 Hollingworth v. Perry (2013); Obergefell v. Hodges (2015).

${ }^{13}$ Elane Photography $v$. Willock pour le refus de photographier un mariage homosexuel considéré comme illégal par la Cour suprême du Nouveau Mexique (2013), et le refus subséquent de la Cour suprême des Etats-Unis de considérer le cas.
} 
exemptent d'obligation d'assurance pour motifs religieux tout employeur institutionnel religieux, qui exemptent de couverture contraceptive toutes les organisations religieuses sans but lucratif et, enfin, qui protègent la liberté de conscience religieuse en rendant obligatoire la disponibilité d'un plan santé sans prise en charge de l'avortement, n'a pas suffi. Par jurisprudence interposée, la Cour suprême a justifié et abondé dans le sens de la posture des républicains redevenus majoritaires au Congrès qui dénonçaient cette réforme comme religieusement liberticide. Ainsi, la Cour suprême par les arrêts Burwell v. Hobby Lobby Stores et Hobby Lobby Stores v. Sebelius de 2014 a reconnu aux entreprises à but lucratif le droit d'être incluses comme 'bénéficiaires' des dispositions prévues par le Religious Freedom Restoration Act, à côté des personnes individuelles et des groupes de personnes religieuses. Elle a accepté l'argument d'une entrave à la liberté religieuse avancé par leurs propriétaires.

La revendication d'exemption a également touché la reconnaissance légale des homosexuels. Elle peut se résumer de la sorte. Les chrétiens revendiquent comme droit de leur conscience la possibilité de refuser d'appliquer la loi - en l'occurrence l'égalité des droits pour les personnes homosexuelles au lieu de leur juste discrimination - qui porte atteinte à leur moralité religieuse. A côté de la bataille du contraceptive mandate, la reconnaissance du couple homosexuel et l'ouverture des législations des États au mariage homosexuel a donné lieu à une intense bataille législative au niveau du Congrès et des États.

Le Congrès a ainsi bloqué la loi fédérale qui prévoyait d'élargir la protection contre la discrimination des LGBT au travail, l'Employment Non Discrimination Act voté par le Sénat en 2013. Ce blocage a obligé Barack Obama à décréter un ordre exécutif en 2014 pour les LGBT employés dans les services fédéraux. Après ce décret, une dizaine d'États dont l'Indiana et l'Arkansas en 2015, ont mis en place des lois équivalentes à la loi fédérale Religious Freedom 
Restoration Act de 1993 pour empêcher la moindre loi locale contre la discrimination homosexuelle dans le travail. Après ce décret également, les républicains ont réintroduit au Congrès en 2015 un projet de loi intitulé First Amendment Defense Act ou FADA, qui a échoué. Il visait à empêcher le gouvernement fédéral de pénaliser une personne refusant d'obéir à une loi parce qu'elle croit que le mariage est, ou devrait être, reconnu comme l'union d'un homme et une femme et que les relations sexuelles sont correctement réservées à un tel mariage. Ce projet donnait littéralement le droit aux employeurs ainsi qu'aux propriétaires de biens locatifs de discriminer pour raison religieuse leurs recrutements et leurs clientèles. Trump a aussi promis l'adoption du FADA et le projet a été réintroduit devant le Sénat en mars 2018. Les versions étatiques du FADA ont pour l'instant été stoppées par des vetos de gouverneurs ou par la justice.

\section{L'éthos public défendu par les démocrates : E Pluribus Unum}

La bataille des républicains s'est finalement portée sur les nominations des juges à la Cour suprême, qui ont fini par devenir majoritairement conservateurs grâce aux nominations de Donald Trump. L'enjeu de ces nominations, à la lumière de ce qui précède, est de fabriquer des jurisprudences qui admettront le droit d'objection de conscience et d'exemption religieuse en cas de discrimination de l'homosexualité. Elles renverseront ensuite l'interprétation libérale des clauses $\mathrm{du} 14^{\mathrm{e}}$ amendement - equal protection et due process - pour limiter encore davantage, si ce n'est mettre fin, à la tolérance légale de l'avortement.

\section{Défense de la séparation}

Face à cette pression constante, cohérente et déterminée, les démocrates défendent une autre conception de l'esprit des lois. Cette conception est globalement «jeffersonienne », axée sur la promotion des droits et libertés et la 
certitude que la finalité de la Constitution est bien de permettre un système qui ne sert qu'à les défendre. Religieusement, ce système protège la diversité religieuse des citoyens, en se "séparant» de la moindre religion établie. Les démocrates ont sécularisé leur compréhension de la séparation, dans une version laïque de la neutralité publique qui est devenue dominante depuis les années 1960. Ils ont récupéré la vision des libres-penseurs issus eux-mêmes des sociétés déistes de la première moitié du XIXe siècle, qui défendaient une secular society basée sur la science, la raison, les valeurs humanistes et le principe de séparation. Plus d'un siècle plus tard, des groupes alors constitués comme les American Atheists, l'Atheist Alliance of America, l'Institute for Humanist Studies et la Secular Student Alliance continuent de défendre la séparation. Ils ont été rejoints par de nouvelles associations, à l'origine de l'affaire Newdow citée plus haut, et militent désormais contre le sectarisme public. Sous le deuxième mandat d'Obama, ils ont contribué à ce que les démocrates déposent un amendement à la loi fédérale sur la liberté religieuse internationale votée en 1998 (IRFA), incluant les athées et les sans-religion déclarés être dans le périmètre de sa vigilance. Cette loi amendée fut la dernière à être signée par Obama en décembre 2016.

La défense de l'éducation publique et, depuis l'ère Roosevelt, celle d'une politique sanitaire et sociale publiques sont deux autres spécificités démocrates. Pendant la période qui nous occupe, deux grandes causes ont mobilisé les groupes défendant le service public : le maintien de l'enseignement de l'évolution dans les écoles publiques, remis en cause dans des centaines d'affaires judiciaires entre 1970 et 2005 (Chélini-Pont 2011), et la préservation des fonds publics pour les écoles, les programmes sociaux et caritatifs publics, au lieu de leur transfert aux associations religieuses et Églises, dans les lois fédérales de 2002 et 2004. 


\section{Protection des droits}

Par ailleurs, en tant que gardiens de la primauté des droits et libertés comme socle de l'ordre public américain, les démocrates ont défendu les droits des femmes. Ils se sont battus pour 1'Equal Rights amendment des années 1970. Ils ont constamment protégé le droit déduit par la Cour suprême de l'avortement précoce, en favorisant le passage de lois le dépénalisant dans la plupart des États. Dans la réforme de la sécurité sociale de 2010, promesse de campagne de Barack Obama, les lois fédérales Patient Protection and Affordable Care Act et le Health Care and Education Reconciliation Act, incluent les soins contraceptifs dans la couverture santé des employeurs, ce qui a provoqué un tollé national.

En ce qui concerne l'égalité des droits et libertés des homosexuels, deux projets de loi démocrates, l'Employment Non-Discrimination Act (ENDA) et le Respect of Marriage Act (RFMA) de 2015 ont été soutenus par Bill Clinton et l'administration Obama. Le premier projet voté par le Sénat a été bloqué à la Chambre et le deuxième a été finalement rendu caduc par les conclusions de l'arrêt Obergefell $v$. Hodges qui considèrent le titre 3 de la loi fédérale Defense of Marriage Act (DOMA) comme inconstitutionnel. Un nouveau projet a été déposé en 2017 à la Chambre des représentants, l'Equality Act. Son introduction a été signée par 241 représentants. Elle fournirait aux personnes LGBT des protections cohérentes et explicites contre la discrimination, dans tous les domaines de la vie : emploi, logement, crédit, éducation, espaces et services publics, programmes financés par le gouvernement fédéral et service de jurés. Elle modifierait la loi sur le logement équitable, la loi sur l'égalité des chances en matière de crédit, la loi sur la sélection des jurés. Elle modifierait également la loi sur les droits civils de 1964 pour interdire la discrimination dans les espaces et services publics et les programmes financés par le gouvernement fédéral sur la base de l'orientation sexuelle. Ce projet de loi sera l'une des batailles du $116^{\mathrm{e}}$ Congrès. 


\section{Promotion du pluralisme religieux et protection des minoritaires}

Enfin les démocrates ont conservé des intuitions de James Madison : la peur de la tyrannie majoritaire et la protection des 'factions' ou groupes minoritaires composant la société. Un tel soutien au pluralisme social comme moteur démocratique a fait leur force électorale. Les démocrates sont ainsi les défenseurs naturels des minorités ethniques, mais aussi religieuses et maintenant linguistiques - les trois se combinant fréquemment - comme résultantes du melting-pot migratoire. Défenseurs des droits civils - hors les démocrates sudistes des années 1960 -, défenseurs de la déségrégation raciale, de la discrimination positive, de la liberté religieuse des juifs et des catholiques quand ceux-ci étaient considérés comme des minorités peu fréquentables, les démocrates sont aujourd'hui les plus ouverts au pluralisme religieux et à la liberté religieuse des musulmans, devenus la plus décriée et la plus surveillée des minorités religieuses de l'histoire américaine.

En termes de symbole, l'ère Obama a été riche en mains tendues. Tout d'abord, lors de son adresse présidentielle de janvier 2009, incluse dans le rituel de l'Inauguration Day, le nouveau président Obama, après avoir fait une allusion attendue aux saintes paroles de l'Écriture et à la divine Promesse, déclare en s'adressant à la foule : «Nous sommes une nation de chrétiens et de musulmans, de juifs et d'hindous, de non croyants ». Le traditionnel "Circle of the We» incorpore des dénominations non chrétiennes - parmi lesquelles l'islamique faisant fi du lien fondateur entre imaginaire biblique et narration nationale. Autre symbole, la prise de parole publique de Barack Obama en juin 2010 pendant la querelle sur la construction d'une mosquée proche de Ground Zero à New York. D'importantes personnalités républicaines, comme Sarah Palin ou Newt Gingrich, avaient bruyamment affiché leur opposition au projet. L'AntiDefamation League (ADL), une organisation juive luttant contre l'antisémitisme, 
s'était aussi déclarée contre. A l'occasion du dîner de l'Iftar organisé à Washington par les élites associatives musulmanes, autre mondanité incontournable des Présidents américains depuis George Bush Jr., Barack Obama y a déclaré le droit de chacun à pratiquer sa religion, « autant les musulmans que quiconque ».

Enfin, ce Président a mis en place, après son grand discours du Caire de 2009 auprès des autorités musulmanes d'Égypte, une nouvelle politique internationale de bonnes relations avec les communautés musulmanes, connue sous le nom de Religious Engagement (Gayte 2014).

\section{Conclusion : un futur déphasage}

$\mathrm{Au}$ terme de cette présentation, nous avons vu que les États-Unis ont connu des politiques publiques récentes dans lesquelles la variable religieuse a été utilisée de manière très différenciée. Sans doute ces derniers temps, l'attitude bienveillante des démocrates à l'égard de la minorité musulmane - quand $60 \%$ de l'opinion publique considère l'Islam comme une religion dangereuse - tout comme leur position ouverte envers l'immigration - considérée par eux comme une force de la société américaine ${ }^{14}$ - et encore leur défense de l'environnement, mondialement représentée par un ancien vice-président démocrate, ces trois affichages ont contribué à faire perdre aux démocrates les deux élections fédérales de mi-mandat sous Barack Obama et les élections présidentielles de 2016. Cependant, la conjonction des représentations religieuses-chrétiennes de la République et du peuple américain, qui a permis aux républicains de capter une opinion majoritaire, peut bientôt trouver ses limites dans les changements de la population américaine.

\footnotetext{
14 Barack Obama a tenté sans succès entre 2011 et 2013 de faire voter par le Congrès le Comprehensive Immigration Reform Act, déposé en 2001 et refusé déjà en 2006 et 2007.
} 
Depuis la fin des années 2000, il est en effet devenu difficile de minorer ou de négliger la part croissante des sans-religion au sein de la population, estimée maintenant à $23 \%$, dont $36 \%$ des jeunes âgés de 18 à 29 ans ${ }^{15}$. Cet électorat qui représente la part la plus jeune des électeurs blancs, est devenu assez indifférent à la dimension religieuse de la psyché publique. De même, une tendance forte se dessine dans le vote féminin contre les républicains depuis 2012, au niveau national et au niveau des États ${ }^{16}$, mue par un sentiment général de régression des droits et de discrimination dans le travail. Enfin, la part des latinos dans la population américaine - 55 millions de personnes en 2018 contre 22 en 1990 et 38 en 2002 - s'accélère de manière spectaculaire, comme conséquence de l'immigration et d'une forte natalité17. Une projection officielle du recensement américain prévoit que $49 \%$ des jeunes américains ayant moins de 18 ans seront dès 2020 des Blancs «non hispaniques », une tendance à la baisse qui devrait s'accentuer et passer à $30 \%$ vers $2060^{18}$.

Cet ensemble de mutations démographiques change l'électorat américain. On pourrait penser que le Parti démocrate bénéficie pour longtemps de la conjonction des votes féminins, des sans-religion et des hispaniques catholiques en croissance. Mais les sans-religion montrent une prédisposition aux idées conservatrices et leur sécularisation totale ne touche que les populations blanches. De même l'électorat hispanique, pour l'instant très fermement démocrate, est également plus pratiquant. Cela signifie que pour capter ces

\footnotetext{
${ }_{15}$ Religious Landscape Study, Pew Research Study, 2014. http://www.pewforum.org/religious-landscapestudy/.

${ }^{16}$ Grace Sparks, «It is impossible to ignore the defection of women voters away from the GOP », CNN [en ligne], 11 novembre 2018; https://edition.cnn.com/2018/11/11/politics/women-exit-poll-vote/index.html.

17 «State Population Change: 2010 to 2018 », United States Census Bureau, 19 décembre 2018; https://www.census.gov/library/visualizations/2018/comm/population-change-2010-2018.html

18 «Older People Projected to Outnumber Children for First Time in U.S. History », United States Census Bureau, 6 septembre 2018; https://www.census.gov/newsroom/press-releases/2018/cb18-41-populationprojections.html
} 
«factions » bientôt pivots dans l'électorat, les républicains devraient lâcher leur « identité » chrétienne exclusiviste, plutôt discriminante envers les femmes et les minorités non blanches. Ils vont aussi devoir réviser - après le mandat de Donald Trump - leurs positions et politiques sur l'immigration dangereuse et incontrôlée. De leur côté, les démocrates ne peuvent espérer garder captifs leurs électorats ethnoreligieux minoritaires, si le groupe des latinos devient le premier groupe démographique et si les démocrates continuent d'axer leur combat libéral vers la défense des LGBT, qui laisse cet électorat particulièrement indifférent. Face à l'axe de défense des droits et libertés reproductives et sexuelles, il faudrait aux démocrates introduire une coloration beaucoup plus religieuse à leurs autres grands thèmes distinctifs comme la justice sociale, l'éducation, l'immigration et l'environnement, autant de questions devenues très sensibles pour les populations pratiquantes (Maibach et al. 2015). C'est en tout cas ce que Barack Obama avait déjà fixé comme objectif à son premier mandat en juin 2006, quand il déclarait devant une assemblée chrétienne rassemblée par le magazine Sojourner :

Je pense que nous faisons une erreur quand nous omettons de reconnaître le pouvoir de la foi dans la vie des gens - dans la vie du peuple américain - et je pense qu'il est temps que nous participions à un débat sérieux pour savoir comment réconcilier la foi avec notre démocratie moderne et pluraliste [...]. La majorité des grands réformateurs de l'histoire américaine n'étaient pas seulement motivés par la foi, mais ils utilisaient de manière répétitive le langage de la religion pour défendre leur cause ${ }^{19}$.

\footnotetext{
19 «Keynote at the Call for Renewal, Building A Covenant for A New America Conference », juin 2006; https://www.nytimes.com/2006/06/28/us/politics/2006obamaspeech.html. (traduction de l'auteure).
} 


\section{Bibliographie}

Barb, A. (2018). Entre Dieu et César. Histoire politique des accommodements religieux aux Etats-Unis. Collection Droit et Religions, Aix-en-Provence, PUAM.

Chélini-Pont, B. (2011). «Bouter Darwin hors des classes. La saga judiciaire de l'enseignement de l'évolution à l'école et ses répercussions sur la laïcité scolaire américaine ». Dans P. Portier, M. Veuille, et J.-P. Willaime (dir.), Théorie de l'évolution et religions. Paris, Riveneuve, p. 239-250.

D'Antonio, W. et al. (2013). Religion, Politics, and Polarization: How Religiopolitical Conflict Is Changing Congress and American Democracy. Lanham (MD), Rowman \& Littlefield Publishers.

Fowler, R. B. et al. (2014). Religion and Politics in America. Faith, Culture and Strategic Choices. Boulder (CO), Westview Press.

Gayte, M. (2014). "La religion, outil incontournable de la smart diplomacy de Barack Obama et Hillary Clinton?». Dans M. Kandel et M. QuessardSalvaing (dir.), Les stratégies du smart power américain: redéfinir le leadership dans un monde post-américain. Etudes de l'IRSEM, n² 23, p. 1119-130.

Issaoui, N. (2016). «Les mouvements religieux minoritaires à l'épreuve du droit états-unien : étude des contours fluctuants de la liberté religieuse du XIXème siècle à nos jours ». Thèse de doctorat, Université Bordeaux 3, Département de civilisation américaine.

Maibach, E. et al. (2015). The Francis Effect: How Pope Francis Changed the Conversation about Global Warming. George Mason University and Yale University. Fairfax (VA), George Mason University Center for Climate Change Communication. $\quad$ http://environment.yale.edu/climatecommunication-OFF/files/The Francis Effect.pdf.

Preston, A., B. Schulman, et J. Zelizer (2015). Faithful Republic. Religion and Politics in Modern America. Philadelphie, University of Pennsylvania Press. 